\title{
Vergleich von Schaumstoffbandagen mit Standard- Unterpolsterung in der Komplexen Physikalischen Entstauungstherapie der Phase I des sekundären Armlymphödems - eine prospektive RCT
}

\section{Comparison of foam Bandages and Standard Padding Material in the Phase I of Complete Decongestive Therapy of Secondary Arm Lymphedema - A Prospective RCT}

\author{
Autoren \\ Institute \\ 1 Charité - Universitätsmedizin Berlin, corporate member \\ of Freie Universität Berlin, Humboldt-Universität zu \\ Berlin, and Berlin Institute of Health, Arbeitsbereich \\ Physikalische Medizin, Berlin, Deutschland \\ 2 Charité - Universitätsmedizin Berlin, corporate member \\ of Freie Universität Berlin, Humboldt-Universität zu \\ Berlin, and Berlin Institute of Health, Institut für \\ Biometrie und Klinische Epidemiologie, Berlin, \\ Deutschland
}

Simone Kornappel ${ }^{1}$, Max Emanuel Liebl ${ }^{1}$ (D), Andrea Stroux ${ }^{2}$, Frauke Glöckner ${ }^{1}$, Anett Reißhauer ${ }^{1}$

Schlüsselwörter

Lymphödem, Kompressionstherapie, Komplexe Physikalische, Entstauungstherapie (KPE), Polstermaterial, Unterpolsterung, Schaumstoffbandagen

Key words

Lymphedema, compression therapy, complex decongestive therapy (CDT), padding material, under padding, foam bandages

eingereicht 11.02 .2021

akzeptiert 28.05.2021

online publiziert $\quad 12.07 .2021$

Bibliografie

Phys Med Rehab Kuror 2021; 31: 321-329

DOI 10.1055/a-1520-1972

ISSN 0940-6689

(c) 2021. Thieme. All rights reserved.

Georg Thieme Verlag KG, Rüdigerstraße 14,

70469 Stuttgart, Germany

\author{
Korrespondenzadresse \\ Simone Kornappel \\ Arbeitsbereich Physikalische Medizin \\ Charité - Universitätsmedizin Berlin \\ corporate member of Freie Universität Berlin \\ Humboldt-Universität zu Berlin \\ and Berlin Institute of Health \\ Charitéplatz 1 \\ 10117 Berlin \\ Deutschland \\ simone.kornappel@charite.de
}

\section{ZUSAMMENFASSUNG}

Hintergrund In der Entstauungsphase der Komplexen Physikalischen Entstauungstherapie des Lymphödems (KPE Phase I) werden verschiedene Untermaterialien eingesetzt, um die Anlage der Kurzzugbinden zu stabilisieren und die Haut zu schützen. Eine neuartige Form der Unterpolsterung bieten mit Baumwolle umnähte Schaumstoffbandagen. Deren Therapieeffekte sind jedoch bislang nicht untersucht.

Material und Methoden Prospektive, randomisierte Nichtunterlegenheitsstudie an Proband/innen mit sekundärem Armlymphödem. Die Kontrollgruppe erhielt eine KPE Phase I mit Standardtherapie (Unterpolsterung aus Schlauchverband und Wattebinde), die Interventionsgruppe erhielt die KPE Phase I mit Schaumstoffbandagen (SC). Primärer Endpunkt war das optoelektronisch gemessene Volumen. Zudem wurde die Umfangsreduktion ermittelt und die Patientenperspektive erfragt. Ergebnisse $n=30$ Proband/innen konnten analysiert werden. SC zeigten einen signifikant größeren Effekt in der Volumenreduktion ( $p=0,026$; $95 \% \mathrm{KI}[-207,9 ;+7,4])$. Bei apriori definiertem Äquivalenzbereich von $\pm 50 \mathrm{ml}$ sind SC der Referenztherapie nicht unterlegen. Die Patienten bewerteten die Abnahme des Spannungsgefühls sowie den Tragekomfort bei SC signifikant besser $(p=0,026$ sowie $p=0,003)$. 
Schlussfolgerung Schaumstoffbandagen sind Standardpolstermaterial nicht unterlegen. Sie können in der Entstauungsphase von Lymphödemen als alternatives Unterpolsterungsmaterial eingesetzt werden. Die Daten liefern Hinweise darauf, dass sie die Reduktion des Ödemvolumens im Vergleich mit bislang verwendeten Untermaterialien verbessern können und das Spannungsgefühl durch sie deutlicher reduziert werden kann.

\section{ABSTRACT}

Background In the intensive phase of complex decongestive therapy for lymphedema (CDT Phase I), different padding materials are used underneath short-stretch bandages to avoid skin lesions and slipping. Foam bandages with vertical foam channels offer a novel form of sub-compression under-padding. Their therapeutic effects have not yet been investigated.

Material and methods Prospective, randomized non-inferiority trial in subjects with CDT phase I for treatment of secondary arm lymphedema. The control group received usual care consisting of sub-compression application of stockinettes and cotton-wool bandages, while the intervention group received sub-compression foam bandages (SC). Volume effects were compared between the groups. Circumference reduction effects and the patient's perspective were surveyed as secondary outcomes.

Results $n=30$ subjects could be analyzed. SC showed a significantly greater effect in volume reduction $(p=0.026 ; 95 \% \mathrm{cl}$ $[-207.9 ;+7.4])$. According to the apriori defined equivalence interval of $\pm 50 \mathrm{ml} \mathrm{SC}$ are non-inferior to reference therapy. Patients using $\mathrm{SC}$ reported a significantly greater decrease in their feeling of tension as well as significantly greater wearing comfort $(p=0.026$ and $p=0.003)$.

Conclusions SC are non-inferior to standard padding material. They can be used as an alternative underpadding material in the intensive phase of complex decongestive therapy of lymphedema. The data suggest that they could improve the reduction of volume compared to conventional padding materials and further reduce a patients' feeling of tension.

\section{Hintergrund}

Goldstandard der konservativen Behandlung des Lymphödems ist die Komplexe Physikalische Entstauungstherapie (KPE). Sie besteht aus einer behandlungsintensiven Entstauungsphase, der Phase I, und einer Erhaltungsphase nach erreichter Volumenreduktion, der Phase II. Therapieziele der KPE Phase I sind neben der Reduktion des Ödemvolumens eine Lockerung bzw. Reduzierung von Gewebsfibrosierungen [1]. Die Therapiedauer der KPE Phase I orientiert sich dabei am Befundausmaß und kann Zeiträume zwischen 6 und 18 Tagen in Anspruch nehmen. Die zur Entstauung notwendige Mobilisierung interstitieller Flüssigkeitsansammlungen wird durch tägliche manuelle Lymphdrainage, eine an den jeweiligen Tagesbefund adaptierte Kompressionsbandagierung sowie Bewegungstherapie bewirkt [2,3]. Die Bandagierung erfolgt in aller Regel in einem schichtweisen Aufbau aus einem zu Hautschutzzwecken angelegten Schlauchverband und elastischen Mullbinden für die Bandage freigestellter Einzelstrukturen wie Finger/Zehen. Es folgen Polstermaterial sowie eine kompressionsgenerierende Schicht aus Kurzzugbinden. Die eingesetzte Polsterung, meist in Form von distal nach proximal gewickelter Wattebinden, soll eine gleichmäßige Druckverteilung ermöglichen. Zur Nivellierung unebener Körperoberflächen können zusätzlich Schaumstoffpads eingebracht werden [2,3]. Eine Polsterung mittels solcher Einlagen stellt so in Regionen wie der Ellenbeuge oder Handinnenfläche einen lokal besseren und gleichmäßigeren Kompressionsdruck her [3,4].

Üblicherweise werden als Unterpolsterung Wattebinden aus Baumwolle, Mischfaser oder synthetischem Material genutzt [5, 6]. Diese Polstermaterialien werden regelhaft jedoch nicht wiederverwendet, da sie bei einem Waschvorgang ihre Form verlieren. Inzwischen steht ein Material zur Verfügung, das aus einer im Vergleich zu Wattebinden dickeren Schaumstoffschicht mit vertikal eingenähten Schaumstoffkanälen besteht, waschbar und damit zur Mehrfachanwendung geeignet ist. Für die Extremitätenversorgung sind aus diesem Material vorgefertigte Bandagehilfen in Universal- und Seriengrößen oder nach Maß erhältlich. Daneben steht Material von der Rolle in unterschiedlicher Breite zur Verfügung [5]. Zum Hautschutz ist dieses Material mit einer Baumwollschicht umnäht, so dass der sonst notwendige Schlauchverband entbehrlich wird. Darüber hinaus ist das Material durch die o.g. Schaumstoffkanäle uneben, was durch Mikromassageeffekte eine effektivere Entstauungstherapie ermöglichen soll.

Da bislang keine Studien zum Einfluss dieses neuen Unterpolsterungsmaterials vorlagen, wurde eine Nichtunterlegenheitsstudie bei einer Stichprobe von Patient/innen mit sekundärem Armlymphödem Stadium II - III in der KPE Phase I geplant. Um die Therapieeffekte in der Entstauungsphase der KPE bewerten zu können, war die Hypothese zu testen, dass eine Therapie mit Schaumstoffbandagen gegenüber der konventionellen Therapie mit Wattierung und Schlauchverband keine signifikante Unterlegenheit aufweist.

\section{Material und Methoden}

Es wurde eine Investigator-initiierte, prospektive, randomisierte, kontrollierte Beobachtungsstudie an Patient/innen mit einseitigem sekundären Armlymphödem in den Stadien II und III geplant, bei denen die Indikation zur KPE Phase I bestand. Ein- und Ausschlusskriterien sowie Abbruchkriterien sind in > Tab. 1 dargestellt.

Screening und Rekrutierung der Proband/innen erfolgten über die Sprechstunde einer Hochschulambulanz mit Schwerpunkt Lymphödem. Bei vorliegender Indikation wurde eine KPE der Phase I auf Basis des jeweiligen Befundes ärztlich verordnet. Nach Information und Einverständnis wurden demografische und behandlungsbezogene Daten im Basisassessment erhoben und die Proband/ innen randomisiert der Kontroll- oder Interventionsgruppe zugeteilt. In der Interventionsgruppe kamen bei der KPE Phase I sodann Schaumstoffbandagen zur Anwendung (Juzo SoftCompress, Vertrieb: Julius Zorn $\mathrm{GmbH}$, Aichach). In der Kontrollgruppe wurden übliche Polsterbinden aus Watte verwendet. Alle weiteren Materi- 
- Tab. 1 Ein- und Ausschluss sowie Abbruchkriterien.

\begin{tabular}{|c|c|}
\hline \multirow{8}{*}{$\begin{array}{l}\text { Einschluss- } \\
\text { kriterien }\end{array}$} & Alter $>18$ \\
\hline & Armlymphödem \\
\hline & - sekundär \\
\hline & - einseitig \\
\hline & - Stadium II - III \\
\hline & Indikation KPE Phase I \\
\hline & $\begin{array}{l}\text { Ausreichendes Sprachverständnis und geistige } \\
\text { Gesundheit }\end{array}$ \\
\hline & Teilnahmebereitschaft und informed consent \\
\hline \multirow{5}{*}{$\begin{array}{l}\text { Ausschluss- } \\
\text { kriterien }\end{array}$} & Absolute oder relative Kontraindikationen für eine KPE \\
\hline & Kontraindikationen für Teile einer KPE \\
\hline & $\begin{array}{l}\text { Fortgeschrittene maligne Erkrankung mit erwartbarem } \\
\text { drop out (palliative KPE) }\end{array}$ \\
\hline & Unklare Ödemgenese \\
\hline & Einnahme von Diuretika \\
\hline \multirow{3}{*}{$\begin{array}{l}\text { Abbruch- } \\
\text { kriterien }\end{array}$} & Unverträglichkeit der Kompressionstherapie \\
\hline & Neues Auftreten von Kontraindikationen \\
\hline & Freiwilliges Ausscheiden von Teilnehmern \\
\hline
\end{tabular}

alien und therapeutischen Bestandteile der KPE Phase I waren für beide beobachteten Gruppen gleich. Der von distal nach proximal abnehmende Druckgradient wurde durch die Anlagetechnik der Bandage sowie die stete Tastkontrolle der Therapeut/innen gewährleistet. Daher war es notwendig, dass lediglich ausgebildete Lymphtherapeuten, die im Rahmen einer Bandageprüfung das Können unter Beweis stellen mussten, diese Entstauungstherapie durchführen dürften.

Als primäre Zielvariable wurde die Volumenreduktion definiert. Hierfür wurde das Extremitätenvolumen optoelektronisch vor und nach der KPE Phase I bei gleichen Messhöhen ermittelt (Perometer Typ 400 NT, Software PeroPlus 2000, Pero-System Messgeräte $\mathrm{GmbH}$, Deutschland).

Als sekundäre Zielvariablen wurden die Umfangswerte am Punkt der (eingangs gemessenen) maximalen Umfangsdifferenz zur nicht betroffenen Seite bestimmt und weiterverfolgt. Anhand der jeweiligen Differenz zum Vortag wurde die Umfangsreduktion sowie deren zeitliche Dynamik im Verlauf der Therapie ermittelt. Weitere sekundäre Zielvariablen waren die Veränderung des Spannungsgefühls aus Patientenperspektive sowie der subjektive Behandlungserfolg. Letztere wurden mittels Fragebogen vor und nach der Beobachtungsphase mittels visueller Analogskala nach Hayes (VAS, $100 \mathrm{~mm}$ ) bestimmt [7]. Der Tragekomfort der Schaumstoffbandagen wurde nach der Beobachtungsphase ebenfalls mittels VAS bewertet. Hinsichtlich subjektiver Nebenwirkungen erfolgte eine Abfrage zum Auftreten von Rutschen und Einschnürungen der Bandagierung. Hier wurde die Anzahl der Therapietage mit diesen Nebenwirkungen sowie ein Abwickeln der Bandagierung dokumentiert.

Fallzahlschätzung: Mit einer Gruppengröße von $n=29$ und ausgehend von $50 \mathrm{ml}$ Äquivalenzgrenze, einer erwarteten Verbesserung der Volumenreduktion durch Schaumstoffbandagen im Vergleich zu Wattebinden um $100 \mathrm{ml}$ sowie einer gemeinsamen
Standardabweichung von 200 ml bei der KPE Phase I des Armlymphödems hat ein einseitiger t-Test bei einem alpha-Fehler von 0,025 eine Power von $80 \%$ und die Nullhypothese der Unterlegenheit zu verwerfen. Unter Berücksichtigung von 2 Dropouts pro Gruppe sollten somit $n=31$ Patienten eingeschlossen werden. Die Fallzahlschätzung wurde mit der Software nQuery + nTerim 4.0 am Institut für Biometrie und klinische Epidemiologie der Charité erstellt. Die Randomisierung wurde mittels PASS Software erstellt. Statistische Analysen erfolgten mit der Software SPSS 27.

Datenauswertung: Der primären Auswertung lag die per protocol Population zu Grunde. Demografische und klinische Merkmale der Interventions- und Kontrollgruppe wurden in Abhängigkeit ihrer Skalierungseigenschaften analysiert. Metrische Variablen wurden aufgrund der oft schiefen Verteilung der Daten anhand nichtparametrischer Tests verglichen. Die Auswertung nominalskalierter Daten wurde über Chi-Quadrat-Tests nach Pearson vorgenommen. Die primäre Zielvariable wurde mittels Mann-Whitney-U-Tests für unverbundene Stichproben auf Signifikanz der Gruppenunterschiede der Volumeneffekte untersucht. Für intra-individuelle Vergleiche zwischen den Untersuchungszeitpunkten wurde der Wilcoxon-Test verwendet. Darüber hinaus wurden 95\%-Konfidenzintervalle berechnet und mit der a priori definierten Äquivalenzgrenze in Beziehung gesetzt [8]. Die Baseline-Adjustierung wurde mittels linearer Regressionsanalyse durchgeführt.

Die Studie wurde von der Ethikkommission der Charité - Universitätsmedizin Berlin (Ethik-Votum EA4/087/17) genehmigt. Im Vorfeld erfolgte eine Datenschutzberatung durch den behördlich beauftragten Datenschutzbeauftragten. Die positive votierte Vorgehensweise wurde jederzeit eingehalten. Die Studie wurde vor dem first patient in beim Deutschen Register Klinischer Studien (DRKS) registriert (DRKS00012982). Die Reihenfolge der Darstellung folgt der Gliederung nach CONSORT 2010 [7].

\section{Ergebnisse}

Von 56 gescreenten Patient/innen konnten nach Anwendung der Ein- und Ausschlusskriterien $n=32$ eingeschlossen und $n=30$ analysiert werden. - Abb. 1 stellt dies detailliert als Flussdiagramm dar. Die berechnete Anzahl an Proband/innen konnte nicht erreicht werden. Ausschlüsse nach Randomisierung wurden nicht verzeichnet, zu Studienbeginn fanden 2 Abbrüche aufgrund von Nebenwirkungen (Druckstellen) in der Interventionsgruppe statt. Alle weiteren Proband/innen konnten die Studie protokollgemäß abschließen. Die Rekrutierung und Beobachtung erfolgte im Zeitraum von 06/2017 bis 03/2020.

Die demographischen und klinischen Charakteristika der Studiengruppen werden in $>$ Tab. 2 dargestellt.

\section{Volumenreduktion}

Sowohl in Kontroll- als auch Interventionsgruppe war eine mit jeweils $p<0,001$ signifikante Volumenreduktion zu verzeichnen. In der Interventionsgruppe fiel die Reduktion mit 447,1 $\pm 153,2$ ml stärker als in der Kontrollgruppe aus. Dort wurde eine Reduktion von 346,9 $\pm 124,3 \mathrm{ml}$ erreicht. Die gemittelten Volumina der Kontroll- und Interventionsgruppe vor (t0) und nach (t1) KPE Phase I sind in $>$ Tab. 3 dargestellt. Es ergibt sich ein um 100,3 $\pm 193,4 \mathrm{ml}$ größerer Effekt der Volumenreduktion in der Interventionsgruppe. 


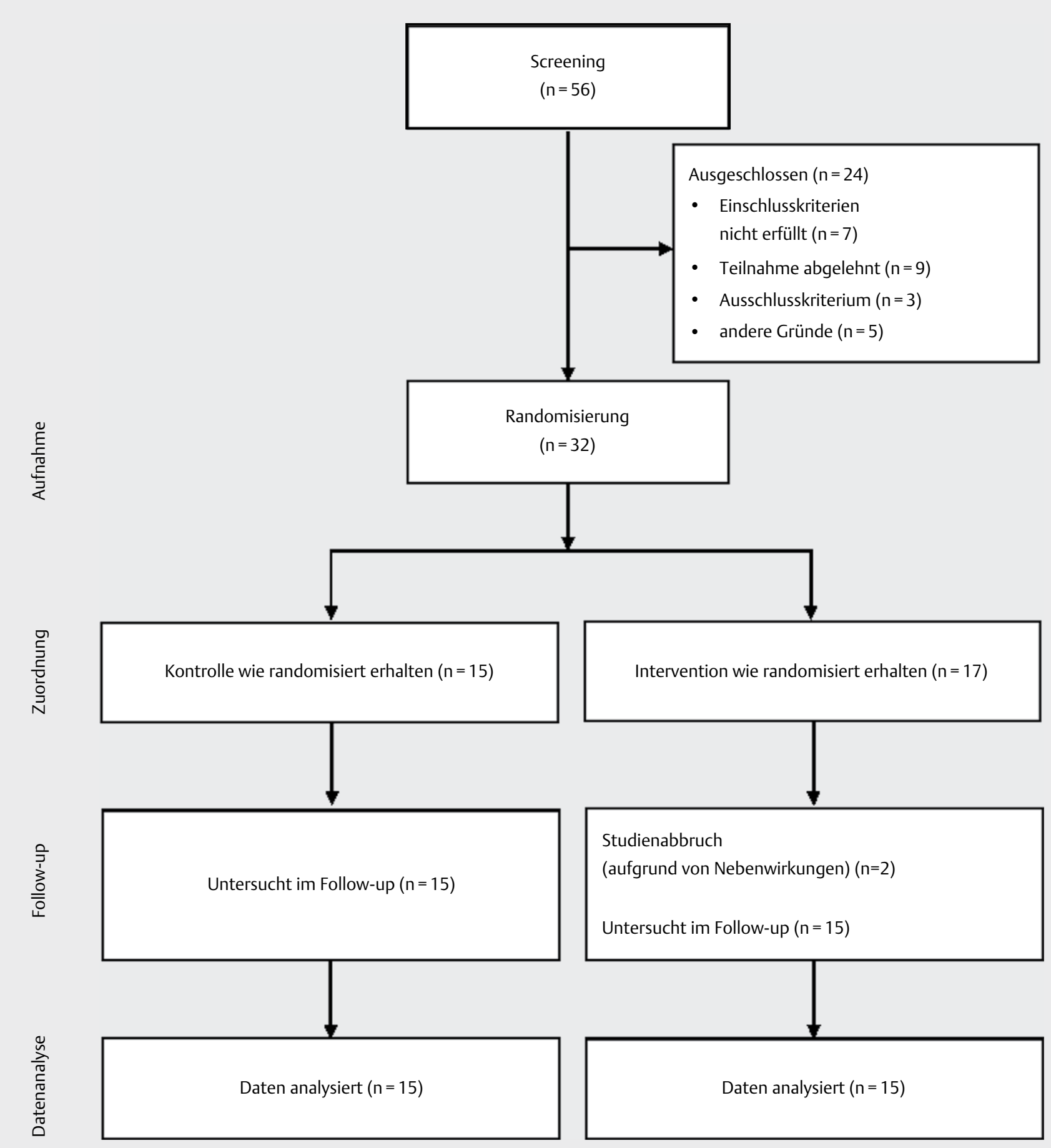

Abb. 1 Flussdiagramm modifiziert nach CONSORT [8].

Der Vergleich beider Volumeneffekte zeigt hier einen signifikanten Unterschied ( $p=0,026$, > Tab. 3). Setzt man die a priori definierte Äquivalenzgrenze von $50 \mathrm{ml}$ und das ermittelte $95 \%$-Konfidenzintervall [-207,9; + 7,4] in Beziehung, so zeigt sich eine Nichtunterlegenheit der Schaumstoffbandagen. > Abb. 2 illustriert dies [8].

Eine Baseline-Adjustierung mittels linearer Regression ergab einen weiterhin positiven Effekt der Intervention, der nicht mehr signifikant war $(p=0,133)$, aber die Hypothese der Nichtunterlegenheit weiterhin stützt.
Nach konservativer LOCF-Imputation (i. e. Baseline-Werte wurden für die 2 fehlenden Follow-up-Werte in der Interventionsgruppe imputiert) für eine zusätzliche Intention-to-treat-Analyse zeigte sich weiterhin eine deutlichere Volumenreduktion (>50 ml) in der Interventionsgruppe um 394,5 $\pm 206,4 \mathrm{ml}$ gegenüber $346,9 \pm 124,3 \mathrm{ml}$ in der Kontrollgruppe, die jedoch nicht mehr signifikant unterschiedlich war $(p=0,156$ bzw. $p=0,171$ nach Baseline-Adjustierung). Die Hypothese der Nichtunterlegenheit konnte damit aber auch in dieser Population gestützt werden. 
> Tab. 2 Demografische und klinische Basisdaten.

\begin{tabular}{|c|c|c|c|c|c|}
\hline & \multicolumn{2}{|c|}{ Intervention $(n=15)$} & \multicolumn{2}{|c|}{ Kontrolle $(n=15)$} & \multirow[t]{2}{*}{ p-Wert } \\
\hline & $\begin{array}{l}\text { Mittelwert } \\
\text { [95\% Konfidenzintervall] }\end{array}$ & Median & $\begin{array}{l}\text { Mittelwert } \\
\text { [95\% Konfidenzintervall] }\end{array}$ & Median & \\
\hline Alter [Jahre] & $58,2[53,2 ; 63,2]$ & 58 & $64,1[56,7 ; 71,5]$ & 65 & 0,202 \\
\hline Erkrankungsdauer [Jahre] & $5,1[3,6 ; 6,6]$ & 6 & $7,5[4,0 ; 11,0]$ & 6 & 0,436 \\
\hline entfernte LK [Zahl] & $12,6[7,9 ; 17,3]$ & 15 & $17,1[11,1 ; 23,2]$ & 18 & 0,267 \\
\hline Größe [cm] & $163,3[157,9 ; 168,7]$ & 161 & $158,3[154,2 ; 162,3]$ & 159 & 0,174 \\
\hline Gewicht t0 [kg] & $80,0[69,2 ; 90,8]$ & 80,8 & $76,3[67,2 ; 85,4]$ & 71,5 & 0,595 \\
\hline BMI $\left[\mathrm{kg} / \mathrm{m}^{2}\right]$ & $29,6[27,0 ; 32,2]$ & 30,4 & $30,6[26,7 ; 34,6]$ & 29,4 & 0,870 \\
\hline \multirow[t]{2}{*}{ Armvolumen initial [ml] } & 5673 [5243; 6103] & 5715 & 5190 [4659; 5721] & 4915 & 0,037 \\
\hline & Anzahl; Prozent & & Anzahl; Prozent & & \\
\hline Geschlecht weiblich & $14 ; 93,3 \%$ & & $15 ; 100 \%$ & & 1,000 \\
\hline Fibrosegrad schwach & $9 ; 60 \%$ & & $7 ; 46,7 \%$ & & 0,715 \\
\hline Fibrosegrad mittel & $6 ; 40 \%$ & & $8 ; 53,3 \%$ & & \\
\hline KKL 2-Versorgung t0 & $14 ; 93,3 \%$ & & $14 ; 93,3 \%$ & & 1,000 \\
\hline KPE Erstversorgung & $8 ; 53,3 \%$ & & $3 ; 20 \%$ & & 0,128 \\
\hline Neoplasie & $14 ; 93,3 \%$ & & $15 ; 100 \%$ & & 1,000 \\
\hline Bestrahlung & $13 ; 86,7 \%$ & & $14 ; 93,3 \%$ & & 1,000 \\
\hline ND Adipositas & $8 ; 53,3 \%$ & & $8 ; 53,3 \%$ & & 1,000 \\
\hline ND Hypertonie & $6 ; 40 \%$ & & $10 ; 66,7 \%$ & & 0,272 \\
\hline ND Schilddrüsenerkrankung & $4 ; 26,7 \%$ & & $3 ; 20 \%$ & & 1,000 \\
\hline ND Kontaktdermatitis & $1 ; 6,7 \%$ & & $0 ; 0 \%$ & & 1,000 \\
\hline ND SLE & $1 ; 6,7 \%$ & & $0 ; 0 \%$ & & 1,000 \\
\hline ND Psoriasis & $1 ; 6,7 \%$ & & $0 ; 0 \%$ & & 1,000 \\
\hline ND Rheumatoide Arthritis & $0 ; 0 \%$ & & $2 ; 13,3 \%$ & & 0,483 \\
\hline
\end{tabular}

- Tab. 3 Volumina der Kontroll- und Interventionsgruppe [ml]. MW= Mittelwert, KI= Konfidenzintervall, STD=Standardabweichung.

\begin{tabular}{|l|l|l|l|}
\hline Volumina & MW [95\% KI] & \pm STD & Median \\
\hline Kontrolle t0 & $5189,9[4658,6 ; 5721,2]$ & $\pm 959,4$ & 4915,0 \\
\hline Kontrolle t1 & $4843,0[4351,1 ; 5334,9]$ & $\pm 888,3$ & 4653,0 \\
\hline Intervention t0 & $5672,9[5243,0 ; 6102,8]$ & $\pm 776,3$ & 5715,0 \\
\hline Intervention t1 & $5225,7[4819,7 ; 5631,8]$ & $\pm 733,3$ & 5014,0 \\
\hline Volumeneffekt Kontrolle (t1-t0) & $-346,9[-278,0 ;-415,7]$ & $\pm 124,3$ & $-327,0$ \\
\hline Volumeneffekt Intervention (t1-t0) & $-447,1[-362,3 ;-532,0]$ & $\pm 153,2$ & $-416,0$ \\
\hline
\end{tabular}

\section{Umfangsreduktion}

Die gemittelten Umfänge der Kontroll- und Interventionsgruppe im Verlauf (an den Therapietagen, d. h. Tage 1 bis 5 sowie 8 bis 12) werden in $>$ Abb. $\mathbf{3}$ vergleichend dargestellt.

In der Interventionsgruppe zeigt sich ein steilerer Abfall der Umfangswerte von Tag 1 zu Tag 2 und von Tag 8 zu Tag 9. Ferner imponiert eine Differenz von Kontroll- und Interventionsgruppe zum Ende der Therapie. Beide Gruppen verzeichnen einen erneuten Umfangsanstieg zwischen Tag 5 und 8 (therapiefreies Wochenende). Die Zahlenwerte dieser Umfangseffekte sind in > Tab. 4 dargestellt.
Ein Vergleich der gemittelten Umfangsreduktionen von Tag 1 zu Tag $2(p<0,001)$, Tag 8 zu Tag $9(p=0,021)$ und Tag 1 zu Tag 12 ( $p=0,021)$ zeigt signifikante Unterschiede zugunsten der Interventionsgruppe. Das Umfangsplus zwischen Tag 5 und 8 (Wochenendeffekt) zeigt keinen signifikanten Gruppenunterschied ( $p=0,486)$. Vergleicht man die Dynamik der Umfangsreduktion wochenweise zwischen den Gruppen, so zeigt sich in Woche 1 kein signifikanter Gruppenunterschied ( $p=0,106)$, für Woche 2 jedoch ein signifikanter Unterschied zugunsten der Interventionsgruppe $(p=0,011)$. 


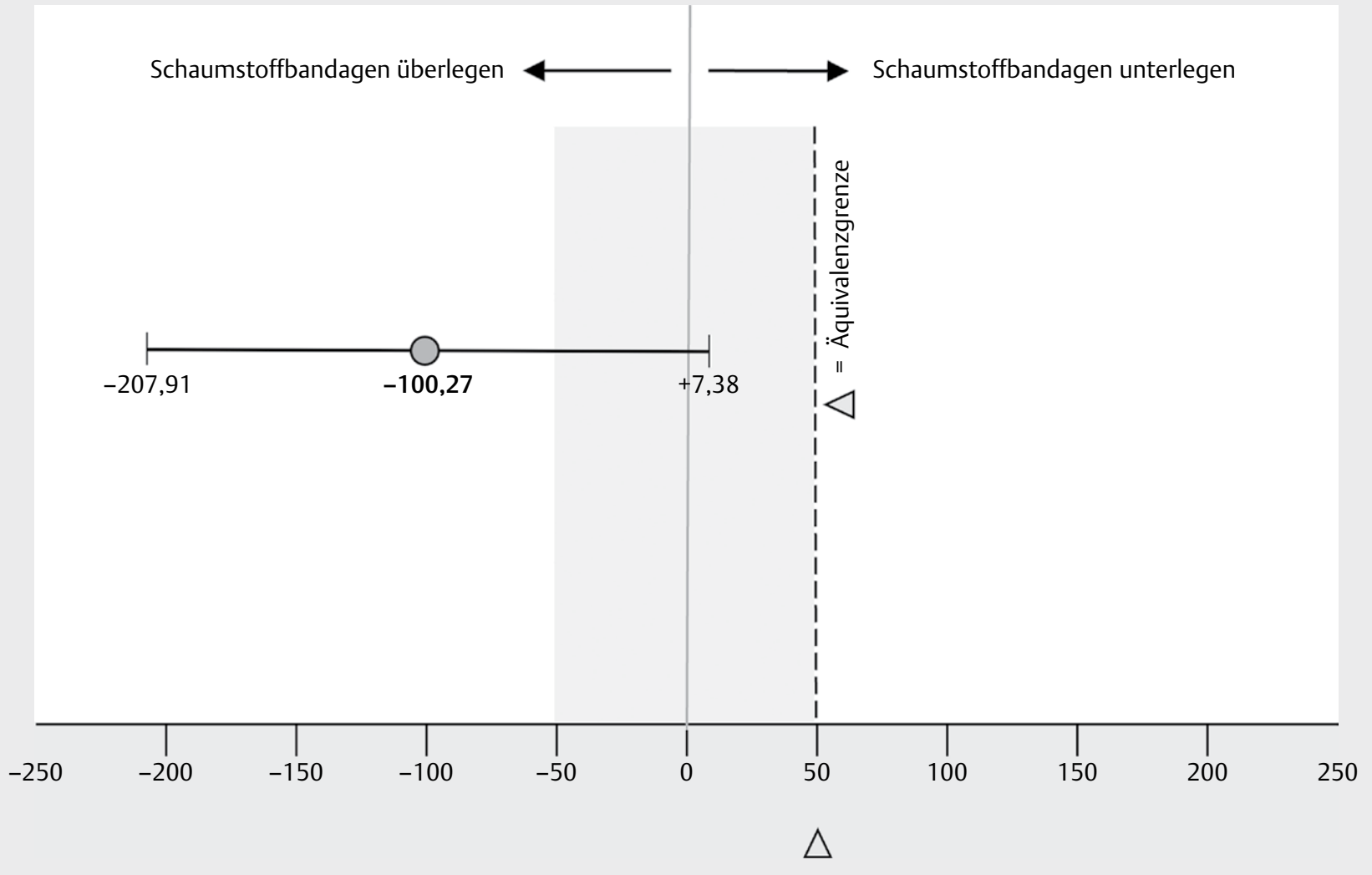

- Abb. 2 95\%-Konfidenzintervall der Differenz der Volumeneffekte in Bezug zur Äquivalenzgrenze (grau unterlegt: apriori definierter Äquivalenzbereich).

\section{Patientenperspektive}

Die durch die Proband/innen mittels 100 mm-VAS vorgenommene Bewertung des Spannungsgefühls belief sich in der Kontrollgruppe zum Zeitpunkt t0 gemittelt auf $47 \pm 20 \mathrm{~mm}$, zum Zeitpunkt t1 auf $22 \pm 18 \mathrm{~mm}$. Das gemittelte Spannungsgefühl in der Interventionsgruppe betrug $60 \pm 22 \mathrm{~mm}$ zum Zeitpunkt t0 und $19 \pm 18$ mm zum Zeitpunkt t1. Im Mittel ergibt sich eine Abnahme des Spannungsgefühls um $25 \pm 11$ mm (53,2\%) in der Kontrollgruppe sowie um $41 \pm 22 \mathrm{~mm}(68,8 \%)$ in der Interventionsgruppe. Der Tragekomfort wird in der Kontrollgruppe im Mittel mit $41 \pm 17 \mathrm{~mm}$, in der Interventionsgruppe mit $61 \pm 18 \mathrm{~mm}$ bewertet. $>$ Abb. 4 und $\mathbf{5}$ stellen diese Ergebnisse dar. Im Vergleich mit Standardpolstermaterial werden Abnahme des Spannungsgefühls sowie Tragekomfort unter Verwendung von Schaumstoffbandagen signifikant besser bewertet $(p=0.026$ sowie $p=0.003)$.

Die Ergebnisse der Abfrage zum Auftreten von Rutschen und Einschnürungen der Bandagierung zeigten signifikant weniger berichtete Tage mit Einschnürungen in der Interventionsgruppe. Gemittelt wurde in der Kontrollgruppe an 1,4 $\pm 1,1$ Tagen ein Einschnürungsgefühl berichtet, in der Interventionsgruppe an $0,7 \pm 1,2$ Tagen ( $p=0.033$ ). Die Anzahl der Tage, an denen ein Rutschen der Bandagierung angegeben wurde, unterschied sich nicht signifikant $(p=0.187)$. Hinsichtlich des vorzeitigen Abwickelns der Bandagierung aufgrund von Rutschen ( $p=0.967$ ) oder Einschnürungen $(p=0.148)$ zeigten sich ebenso keine signifikanten Gruppenunterschiede.

\section{Adverse Events}

Es kam zu 2 Dropouts mit Abbruch des Studienprotokolls aufgrund von vermuteten bzw. möglichen Therapienebenwirkungen. Es wurde in beiden Fällen einmalige Einschnürungen an der Außenseite des fünften Fingers beobachtet, die am ehesten durch Druckeffekte bei Materialüberlappung oder behandlerbedingt verursacht wurden. In beiden Fällen konnte die KPE außerhalb der Studie fortgeführt werden, die Symptome waren schnell und vollständig rückläufig.

\section{Diskussion}

\section{Limitationen}

Aufgrund der seit Anwendung der Sentineltechnik stetig sinkenden Inzidenz des sekundären Armlymphödems nach Mammakarzinom [9-13] sowie dem Stopp der Präsenzforschung in der Corona-Pandemie konnte die per Fallzahlschätzung berechnete Anzahl an Proband/innen nicht erfüllt werden. Damit liegt den signifikanten Ergebnissen und der statistisch belegten Nichtunterlegenheit lediglich eine kleine Stichprobengröße zugrunde.

Ferner ergab sich trotz durchgeführter Randomisierung ein signifikant höheres initiales Armvolumen in der Interventionsgruppe. Obschon sich beide Gruppen hinsichtlich BMI und Gewicht nicht signifikant unterscheiden, zeigen diese volumenassoziierten Parameter in der Interventionsgruppe im Median bereits höhere Werte. 


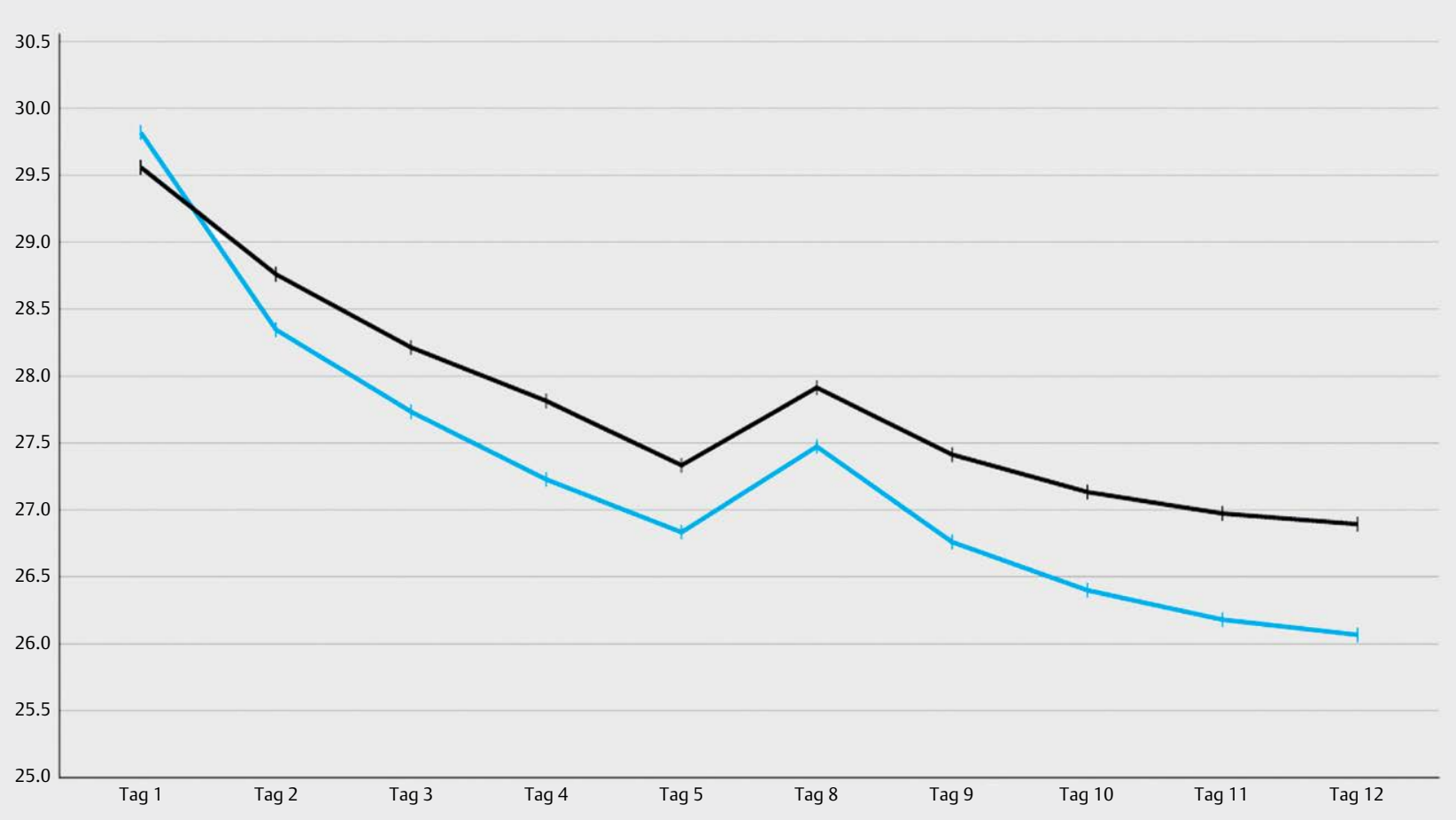

Abb. 3 Umfangsmittelwerte der Kontroll- und Interventionsgruppe im Verlauf (schwarz: Kontrollgruppe, blau: Interventionsgruppe).

> Tab. 4 Umfangseffekte der Kontroll- und Interventionsgruppe [cm]. MW= Mittelwert, STD=Standardabweichung.

\begin{tabular}{|c|c|c|c|c|}
\hline \multirow{2}{*}{ Umfangseffekte } & \multicolumn{2}{|c|}{ Intervention } & \multicolumn{2}{|c|}{ Kontrolle } \\
\hline & MW [95\% KI] & \pm STD & MW [95\% KI] & \pm STD \\
\hline Umfangseffekt Tag 2 - Tag 1 & $-1,5[-1,2 ;-1,8]$ & $\pm 0,5$ & $-0,8[-0,7 ;-0,9]$ & $\pm 0,2$ \\
\hline Umfangseffekt Tag 9 - Tag 8 & $-0,7[-0,5 ;-0,9]$ & $\pm 0,3$ & $-0,5[-0,3 ;-0,7]$ & $\pm 0,4$ \\
\hline Umfangseffekt Tag 12 - Tag 1 & $-3,8[-3,1 ;-4,4]$ & $\pm 1,2$ & $-2,7[-2,3 ;-3,0]$ & $\pm 0,6$ \\
\hline Umfangseffekt Tag 8 - Tag 5 & $0,6[0,5 ; 0,7]$ & $\pm 0,2$ & $0,6[0,5 ; 0,6]$ & $\pm 0,1$ \\
\hline Umfangseffekt/d (Woche 1) & $-0,7[-0,6 ;-0,9]$ & $\pm 0,3$ & $-0,6[-0,5 ;-0,6]$ & $\pm 0,1$ \\
\hline Umfangseffekt/d (Woche 2) & $-0,4[-0,3 ;-0,4]$ & $\pm 0,1$ & $-0,3[-0,2 ;-0,3]$ & $\pm 0,1$ \\
\hline
\end{tabular}

Wiederum nicht signifikant, aber in diesem Zusammenhang ebenfalls zu diskutieren, ist der höhere Anteil von Proband/innen mit KPE-Erstverordnung in der Interventionsgruppe (53 vs. $20 \%$ ). Ein potenzieller Einfluss auf Volumen- und Umfangseffekte kann auch hier nicht ausgeschlossen werden, auch wenn sich beide Gruppen hinsichtlich des klinisch beurteilten Fibrosierungsausmaßes nicht unterschieden.

\section{Diskussion der Hauptergebnisse}

Diese Studie ist nach Kenntnis der Autor/innen die erste randomisiert-kontrollierte Studie, die die Anwendung von Schaumstoffbandagen im Vergleich mit Standard-Unterpolsterungsmaterial in der Entstauungsphase der KPE untersucht. Die Studie war auf Nichtunterlegenheit ausgelegt. Die Nichtunterlegenheit der Schaumstoffbandagen konnte belegt werden. Hinsichtlich der Volumeneffekte zeigten die Schaumstoffbandagen eine effektivere, statistisch signifikante Volumenabnahme im Beobachtungszeitraum. Als Wirkhypothese wird ein Mikromassageeffekt des Materials aufgrund veränderter Druckverteilungen durch unebene Oberflächen postuliert. Weitere Studien, die diese Annahme mittels In-vivo-Druckmessungen unter der Kompressionsbandagierung untersuchen, sind empfehlenswert $[6,14]$.

Eine Überlegenheit von Schaumstoffbandagen lässt sich aufgrund des gewählten Studiendesigns nicht formulieren. Gleichwohl legen die Ergebnisse nahe, dass eine Tendenz zur Überlegenheit der Schaumstoffbandagen gegenüber der konventionellen Therapie mit Wattierung und Schlauchverband bestehen könnte. Hierzu wären weitere Untersuchungen mit einem entsprechend ausgelegten Design sinnvoll. 


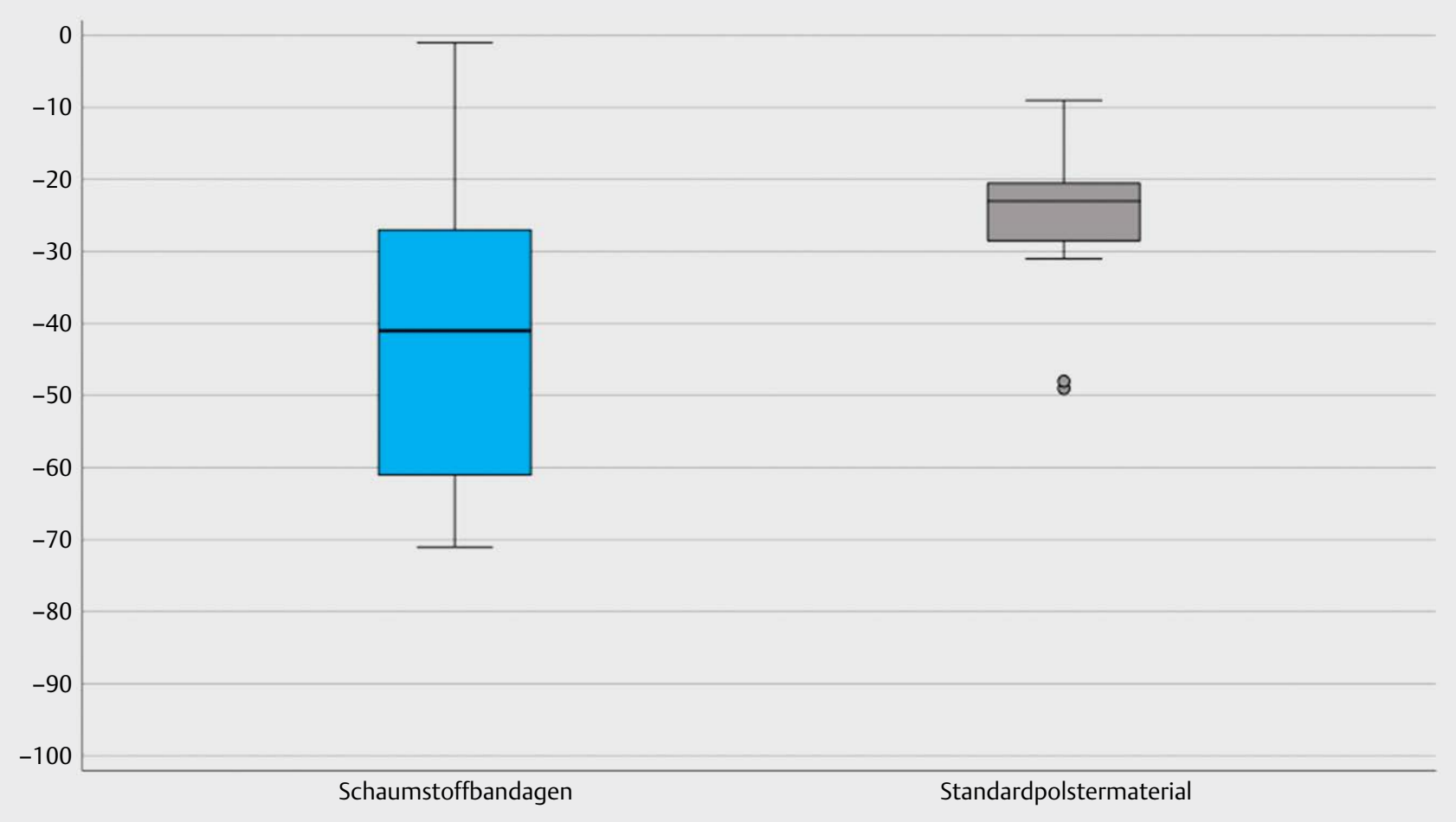

- Abb. 4 Abnahme des Spannungsgefühls (Differenz t0-t1) in Kontroll- und Interventionsgruppe. Boxplots. Y-Achse: Abnahme in mm VAS.

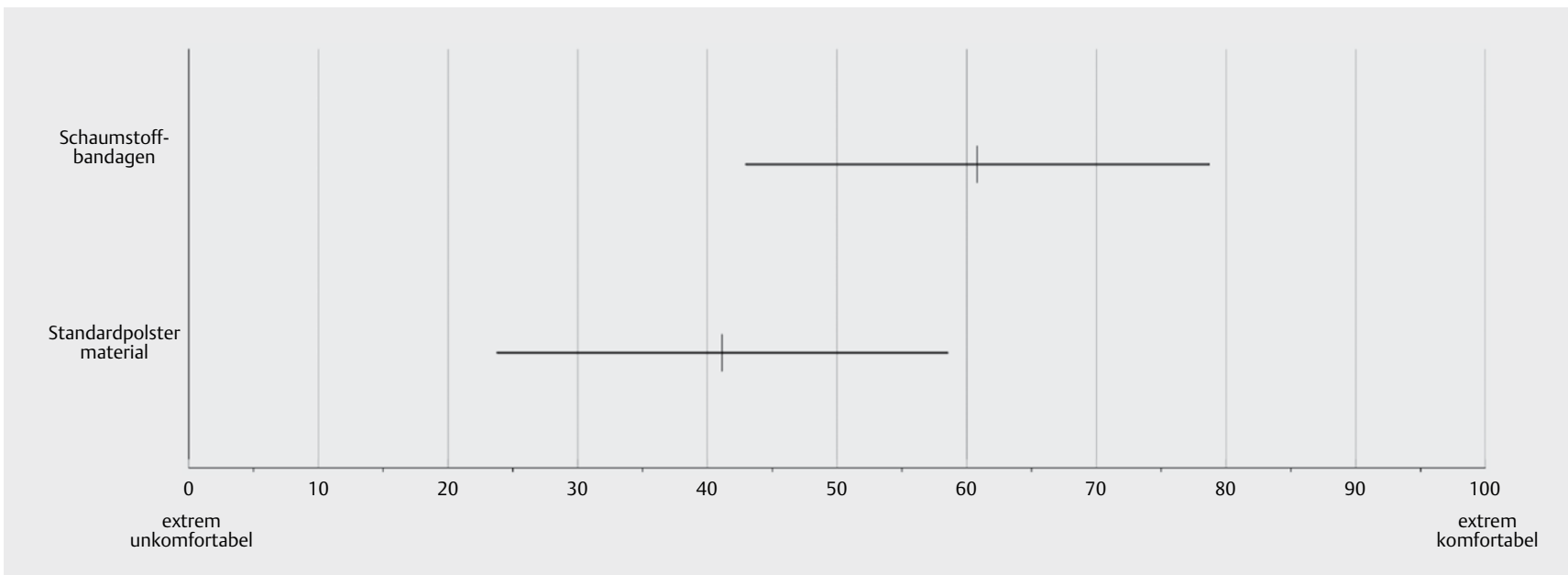

Abb. 5 Tragekomfort im Vergleich. VAS: 0 = extrem unkomfortabel, 100 = extrem komfortabel; Mittelwert und Standradabweichung.

In puncto Umfangsreduktion konnte gezeigt werden, dass sich unter Anwendung von Schaumstoffbandagen signifikant stärkere Reduktionseffekte direkt zu Therapiebeginn und nach der Wochenend-bedingten Therapiepause ergeben und dass es mit diesem Material zu einem schnelleren Erreichen erster Therapieeffekte kommt. Zudem ist bei wochenweiser Betrachtung im Gruppenvergleich in der zweiten Therapiewoche eine signifikant effektivere Umfangsreduktion in der Interventionsgruppe gezeigt worden. Das erneute Umfangsplus in beiden Gruppen über die Wochenenden ohne Kompressionstherapie sollte als Teilergebnis besonders hervorgehoben werden und stützt die klinischen Beobachtungen na- hezu jeder KPE I. Die Verwendung anderer, auch neuer Materialien, wie Medizinische Adaptive Kompressionssysteme (MAK) sollte in diesem Zusammenhang diskutiert werden, um Therapieerfolge auch an Wochenenden oder bei Therapieausfällen aufrecht erhalten zu können [2, 14-16].

Überdies zeigten sich signifikant positive Effekte der Schaumstoffbandagen hinsichtlich Tragekomfort und der Abnahme des Spannungsgefühls sowie ein signifikant weniger häufig berichtetes Einschnürungsgefühl unter Verwendung der Schaumstoffbandagen. Auch diese Effekte lassen sich auf differente Materialeigenschaften und den postulierten Mikromassageeffekt zurückführen. 
In der Zusammenschau lassen die hier beschriebenen Effekte mögliche Vorteile hinsichtlich der Therapietreue erwarten. Zwar ist dieser Aspekt bislang schwer messbar, ist aber unabdingbare Grundlage einer effizienten und erfolgreichen Entstauungstherapie und dabei von zentraler Bedeutsamkeit in der lebenslangen Behandlung von Lymphödempatient/innen.

\section{Zusammenfassung und Ausblick}

Schaumstoffbandagen können in der Entstauungsphase von Lymphödemen als alternatives Unterpolsterungsmaterial eingesetzt werden und sind Standardpolstermaterial nicht unterlegen. Die vorliegende Studie liefert starke Hinweise dafür, dass ihre Verwendung die Reduktion des Ödemvolumens im Vergleich mit bislang verwendeten Untermaterialien verbessern kann, höhere Fallzahlen in größer angelegten Folgestudien würden die Evidenz dafür erhöhen. Therapieeffekte könnten mit ihrem Einsatz schneller erreicht, das Spannungsgefühl deutlicher reduziert werden.

Es ist sicher lohnend, auch die ökologischen und ökonomischen Aspekte durch die Wiederverwendbarkeit der Schaumstoffbandagen gesondert zu betrachten.

\section{Danksagung}

Die Julius Zorn GmbH, Aichach, unterstützte die Studie mit Drittmitteln und stellte das Schaumstoffuntermaterial zur Verfügung.

Interessenkonflikt

AR erhielt zur Durchführung der Studie Drittmittel und Material seitens der Julius Zorn GmbH, Aichach. AR, ML und SK erhielten Referentenhonorare der Juzo $\mathrm{GmbH}$.

\section{Literatur}

[1] AWMF. S2k Leitlinie Diagnostik und Therapie der Lymphödeme.AWMF Reg.-Nr. 058-001. https://www.awmf.org/uploads/tx_szleitlinien/058001I_S2k_Diagnostik_und_Therapie_der_Lymphoedeme_2019-07.pdf Letzter Aufruf: 27.11.2020

[2] AWMF. S2k Leitlinie Medizinische Kompressionstherapie der Extremitäten mit Medizinischem Kompressionsstrumpf (MKS), Phlebologischem Kompressionsverband (PKV) und Medizinischen adaptiven Kompressionssystemen (MAK). AWMF-Reg.-Nr. 037/005 https://www.awmf.org/uploads/tx_szleitlinien/037-005I_S3k_Medizinische-Kompressionstherapie-MKS-PKV_2019-05.pdf (Letzter Aufruf: 13.01.2021)
[3] Reißhauer A, Auler S, Bieringer B, Jahr S Physikalische Entstauungstherapie. In: Reißhauer A et al., (Hrsg.). Kompendium der lymphologischen Kompressionsversorgung. Berufsfachschule für OrthopädieTechnik 2009; pp. 42-57

[4] Asmussen P, Strößenreuther RHK. Kompressionstherapie. In: Földi E, Földi M Hrsg. Lehrbuch Lymphologie für Ärzte, Physiotherapeuten und Masseure/med. Bademeister. 7. Aufl. Stuttgart: Urban \& Fischer; 2010: 511-572

[5] Bringezu G, Schreiner O. Bandagierung. In: Bringezu G, Schreiner O, Hrsg. Lehrbuch der Entstauungstherapie. 4. Aufl. Springer-Verlag; 2014: 173-177

[6] Protz K, Reich-Schupke S, Müller K et al. Kompressionsverbände mit und ohne Unterpolsterung. Hautarzt 69: 2018; 653-661

[7] Hayes MH. Experimental developement of the graphics rating method. Physiol Bull 1921; 18: 98-99

[8] Piaggio G, Elbourne D, Pocock S et al. Reporting of Noninferiority and Equivalence Randomized Trials. Extension of the CONSORT 2010 Statement. JAMA. 2012; 308: 2594-2604

[9] Netopil B. Häufigkeit sekundärer Arm-, Mamma und Thoraxwandödeme nach Mammakarzinomtherapie heutzutage. Eine retrospektive Studie mit 1000 einseitig am Mammakarzinom operierten Patientinnen (mit Erstdiagnose von 2000-2007). Dissertation Universität Gießen. 2010;

[10] Vaezipour N. Inzidenz und Risikofaktoren des sekundären Lymphödems nach Therapie des Mammakarzinoms. Dissertation Universität Freiburg; 2015

[11] GEK-Heil- und Hilfsmittel-Report 2008: Beckermann, M: Die Behandlung eines Lymphödems bei Brustkrebspatientinnen. Asgard-Verlag; Band 64, S 49-74

[12] Herpertz U. Ödeme und Lymphdrainage.Schattauer-Verlag; Stuttgart: 4. Auflage 2010: S 139

[13] McLaughlin SA, Wright M], Morris KT et al. Prevalence of Lymphedema in Women with Breast Cancer 5 Years After Sentinel Lymph Node Biopsy or Axillary Dissection: Patient Perceptions and Precautionary Behaviors. J Clin Oncol 2008: 26; 5220-5226

[14] Reißhauer A, Kornappel S, Tsatrafilis E et al. Medizinische Adaptive Kompressionssysteme sind Flachstrick-Kompressionsstrümpfen in der KPE Phase II nicht unterlegen. Physikalische Medizin, Rehabilitationsmedizin, Kurortmedizin 2020; 30: 214-221. doi:10.1055/a-1097-7675

[15] Damstra RJ, Partsch H. Prospective, randomized, controlled trial comparing the effectiveness of adjustable compression Velcro wraps versus inelastic multicomponent compression bandages in the initial treatment of leg lymphedema. J Vasc Surg Venous Lymphat Disord 2013; 1: 13-19

[16] Mosti G, Cavezzi A, Partsch H et al. Adjustable Velcro Compression Devices are More Effective than Inelastic Bandages in Reducing Venous Edema in the Initial Treatment Phase: A Randomized Controlled Trial. Eur J Vasc Endovasc Surg 2015; 50: 368-374 